perfectly healthy subjects. A similar remark applies to the vulva of females.

We next turn to the secretion from the kidneys, and find that it is no exception to the rule. The secretion is increased by fear-the most depressing of all our passions; by hysteriaessentially a disease of debility; and in diabetes insipidus, which, there is reason to believe, is the result of extraordinary debility of the constitution, rather than its cause, inasmuch as the amount of solid matter excreted is too small in itself to produce any marked effect on the system. Few are ignorant how much the secretion of the bladder is increased during that low state of vitality which accompanies paraplegia; and how the mucus, till then hardly recognised in the urine, becomes one of its most characteristic ingredients.

Respecting the liver, I have not much definite information to offer: all we can say is, that its sugar-production is increased, as in diabetes, by certain debilitating agencies, and is diminished by such things as increase the patient's strength. Respecting the influence of debility in increasing the secretion of the stomach, we cannot say much beyond the fact that waterbrash is common in those whose stomachs are weakened by ulceration, etc.

But our facts do not end even here; for we may now turn to parts in which the amount of secretion is naturally so small as to be imperceptible, viz., the eye and external ear. In the former, we have increased secretion in the poorly fed, the delicate, the strumous, and the aged. Shakespeare, amongst the characters of old age, sets down "a moist eye", "eyes purging thick amber and plum.tree gum." And we may add as a significant fact, that those who are weakly, not only weep sooner, but cry with far more copious tears than those who are strong; that an hysterical burst of crying may be repeatedly checked by a glass of wine, or other stimulant; and that many a man will, when fasting, weep over griefs, which cannot bring a single tear if presented to him when full. In the ear, we have otorrhen occurring amongst the scrofulous, the children of consumptive patients, and in those who are or have been reduced by scarlatina or other depressing diseases. I have seen it come on when the patient was at the lowest extremity of wealness, and go off as he returned to his strength.

We speak with some hesitation respecting the influence of deficient vital power on the secretion of the milk in the female. But we think that we have noticed, as a general rule, that very healthy women have not so large a quantity as the weakly; that the quantity in the weakly increases in proportion to the debility lactation produces in them; that large quantity and good quality do not generally go together; and that the in. fluence of tonics and stimuli upon a nurse, is to increase her own health and condition, and to diminish the quantity of the milk produced.

Let us for one moment turn to one of the causes of increased secretion, the presence of so-called chronic inflammation. What are the facts connected therewith? why, that during the acute stage of the disease, when the power or increased action may be considered at its height, all secretion is suspended and the secreting surfaces are dry; that the increased secretion comes on when the inflammation is cinronic, and that the parts are restored to health under the influence of stimulants. We know how much debility, locally and generally, increases the secretion of pus from a sore; how it abounds under the use of poultices, and almost disappears when dry lint is used.

Coupling all these facts together, we are forced to the conclusion, that increase in the amount of any secretion, is not cvidence of increased action or augmented power, but that it may, and very frequently does, result from diminished vitality, and even expiring life. How frequently these last conditions are the cause I would hardly venture to enunciate. My im. pression is, that they are far more common than any one has hitherto supposed.

The practical application of the view we have been setting forward is readily appreciable. Chronic bronchitis, strumous coryza, and some other profuse discharges, have long been treated by stimulants and the most generous diet; but in some other diseases, which fairly come into a similar caterory, the same principles are studiously avoided. Thus, in gonorrhœa, while we give with one hand such stimulants as copaiba and cubebs, and even apply such local irritants as cantharides ex. ternally, we take away with the other hand the patient's beer and wine. I have long thought that this plan could not be the correct one, and I have sought, first, for some reason why alcoholic stimuli should be prohibited in any case; secondly; when they might be administered with advantage. The reason why they are in general tabooed is, because gonorrhoa is most common in young men, that it prevents them from having cosnection, that this encourages erections, that erections are very painful, and that alcoholic stimuli increase sensual desire, and promote erections-hence they are forbidden. But if erections are not troublesome and rarely, if ever, occur; if the patient is not strong, is of a strumous habit, has much exertion, or suffers from any cause of debility, tonics and alcoholic stimuli may be given with great advantage. Under their use I have seen cases of gonorrhœa yield rapidly, which had previous!y appeared to be intractable.

But there is another application of the sulject to which I must refer in a few words. If the increased secretion into the cells of the areolar tissue in general dropsy has its origin in an enfeebled condition of the system (and we will not waste time by demonstrating that it is not produced simply by obstruction to the circulation, for that will exist as in phlegmasia dolens, etc., without dropsical effusion, and heart discase without dropsy is common enough), is not any plan of treatment which ignores the use of strengthening remedies manifestly insuffi. cient to complete the cure? Is not the plan of treating it by diuretics and frequent purgation, alone, radically bad? To these observations I will add, that I have had under my care a girl with general dropsy from renal disease, in which an artificial diarrhoa had been kept up for some months, ere she came into hospital, and during that period the anasarca had steadily increased. On her admission, the treatment was entirely changed, the purging checked, and tonics given, and an improvement was immediately apparent. She remained, however, only under notice for a fortnight, and was taken away by her friends, the dropsy remaining uncured.

I doubt not that many practitioners have seen many mild cases of dropsy cured by tonics alone; but I have already taken up so much space, that I must not pursue the subject farther.

\section{THE MEDIAN OPERATION OF LITHOTOMY, FOR THE REMOVAL OF A PORTION OF BOUGIE FROM THE BLADDER.}

By Augustin Prichard, Esq., Surgeon to the Bristol Royal Infirmary.

ON the morning of Sunday, February 20th, I met two surgeons in consultation, in a case of which I received the following history :-

Mr. A. B. C., aged 28, in good health but of spare halit, on the morning of January 31 st (i.e., three weeks before I saw him), having been under the impression that he had a stricture, passed with some difficulty a No. 10 bougie or gum elastic catheter along the urethra; and, after allowing it to remain in the canal about twenty minutes, he found he could not with draw it. After repeated attempts, by pulling and twisting the instrument, he withdrew it, leaving about three inches behind. He was seen by his ordinary medical attendant; and, in the afternoon, by another surgeon; and they made many attempts to lay hold of the foreign substance with the urethra forceps. They could only succeed in bringing away small portions of the bougie, which appeared to be held by the stricture too firmly to allow of its removal in this way; and, consequently, they placed the patient in a position for lithotomy, and under the influence of chloroform, and with a silver catheter in the urethra, held in contact with the portion of bougie, they cut down immediately behind the scrotum, in the middle line. Upon exposing the end of the bougie, and attempting to withdraw it with the aid of the dressing forceps, after repeated trials, it unfortunately eluded the grasp of the forceps, and slipped back into the bladder. A silver catheter was passed and retained, and a bladder of cold water was applied to the perineum, to stop the hæmorrhage, which was considerable. From this time, for some days, there was but little inconvenience; the patient passed urine every two or three hours. The wound granulated freely and gradually closed; but afterwards the irritability of the bladder increased, and on February the 18th, the wound being almost healed, extravasation of urine took place; the scrotum and perineum became much inflamed, red, swollen, and painful the wound became unhealthy, and the general state of the patient was as bad as could be, so much so, notwithstanding the stimulus prescribed in the morning, that in the night of February 19th, he had excessive rigors, and appeared to be dying.

When I saw him, early on the 20th, he had somewhat ralled; 351 
he looked sallow and collapsed, his pulse was 130 and very feeble, and his scrotum and perineum hard and painful, and evidently on the point of sloughing; and after considering his state, and the probability of his sinking rapidly, if not relieved of the cause of the irritability of the bladder, as well as of the urine in the cellular tissue of the perineum, we determined to operate, and remove the bougie by the median section.

He was placed on a table, and tied up in the usual position for lithotomy; and I proceeded to operate, substituting two or three glasses of brandy and water for the chloroform, as I was fearful of the depressing effect of the anæsthetic, in a man so worn and weak as our patient was. The gentlemen whom I met in consultation, kindly gave way to my wishes in this respect, for I think that they were inclined to administer chloroform, and save the patient the half hour's torture which was inflicted upon him; but now that the case is concluded and the result has turned out as satisfactorily as we could possibiy hope for, advocate as I am for chloroform in the majority of cases, I think that the danger which this gentleman went through would have been considerably increased by the use of that agent, particularly as I believe that on the former occasion he suffered greatly from sickness afterwards, and he appeared to me to be too much like a man likely to sink, to justify us in running any additional risk.

With the staff in the bladder and finger in the rectum, I ran a sharp-pointed knife through the lower part of the old wound and sloughing perineum into the staff, cut upwards so as to enlarge the opening, passed the probe into the bladder, and followed it up by the finger. The prostate felt very much constricted round the point of my finger; and, owing to the infiltration of urine in the perineum and consequent tumefaction, I could not reach readily into the bladder, so as to feel for the portion of bougie, which was, however, readily felt by the forceps.

After repeated trials with two or three kinds of forceps, and after bringing away little bits in the blades of the instrument, it was at last seized about the middle, rotated, and removed The bladder was washed out with warm water, and the patient lifted bnck into his bed; and, although he had undergone nearly half an hour's severe suffering, during which his shrieks must have been heard at a great distance, it was at once obvious that his state was in every respect improved. His pulse had fallen in frequency, his pain had subsided, some of the anxiety of his countenance had disappeared, and, from the free opening of the former wound, and the manipulation the parts had un dergone, the scrotum and perineum had lost their redness and tension by the lealing out of the urine and effused serum; and he expressed himself as relieved and comfortable. A tonic and stimulating plan of treatment was resolved upon.

The nortion of bougie removed consisted of nearly three inches of what seemed to be a gum elastic catheter, dark brown in colour, doubled up and coated with a layer of phosphates, so that it came off in a distinct shell when the forceps laid hold of it.

From this time, for a fortnight, his progress towards recovery was uninterrupted; the wound was closing, and the urine began to How in its natural channel, when a severe attack of diarrhœu, brought on by error of diet, retarded his progress for some days. This yielded to judicious treatment; and, when I last heard, al the irritability of the bladder had disappeared, the urine flowed chiefly through the urethra, and the patient was rapidly regaining his strength.

I did not see him after the day of the operation, and I therefore have to thank the gentlemen who attended him for many of the notes which have enabled me to draw up this case, as well as for their skilful care of the patient.

We have heard this expression used by older surgeons, " the wholesome stimulus of the knife; " and, in this instance, instead of the collapse and shock we generally see after a severe operation, the patient appeared so much roused and relieved, that he was obviously stimulated by the treatment he had undergone.

Lastly, my object in publishing this history is not only for the pleasure of narrating a successful case, but also for the sake of bringing forward what I consider to be a triumphant example of Allarton's operation; for the bougie was, in this instance, removed from the bladder without any injury to the deeper parts, and with no more incision than what was necessary to relieve the infiltrated perineum, and to these conditions, including the power which the patient retained from the first of holding his water properly, the rapid recovery which he made was unloubtedly due; and I think it very questionable whether he could have escaped with his life from the more serious perils of the lateral operation.

\section{Goulstomian apertures}

FEVER AND INFLAMMATION.

DELIVERED BEFORE THE

ROYAL COLLEGE OF PHYSICIANS, LONDON, 1859.

By Wrumant Addrson, M.D., F.R.S., Fellow of the College.

Lectune I (continued).

III.-The Fluid or Ptasua of the Blood,

The plasma is a very complicated fluid. Its chief constituents are water, fibrine, albumen, colourless corpuscles, volatile matter, fatty compounds, and salts. In its passage through the capillary vessels it permeates all organs and tissues; and, at various points of the circulation, the parenchymatous depurating organs remove from it those substances, which, if retained in it, would injure its qualities. We tind that numerous soluble compounds - substances, for instance, which have been taken as food or drink, and poisons received into the stomach-are absorbed into the plasma, whence they may be again eliminated or removed, changed or unchanged by the depurating organsthe organs of secretion.

Many of the common poisons have a local action as irritants, and also a remote or special action upon some parenchymatous organs; other poisons exhibit only the remote or special action. Cantharides has a special action as an irritant to the stomach; it also affects, remotely, through the plasma, the urinary organs. Morphia, taken by the stomach, affects specially the brain, through the plasma. Digitalis, taken by the stomach, affects the heart; strychnia the spinal marrow, through the same medium.

In experiments on animals with prussic acid, it has been found when death took place within a few minutes only, that the odour of the poison could be perceived in the cavities of the body; and again in other experiments, where preparations of antimony were injected into the blood by a vein in the thigh, the metal has been found in the coats of the stomach.

From these and numerous other facts, which it is unnecessary to particularise, we learn that poisonous substances, taken by the stomach, enter the plasma or mingle with the fluid of the blood; and thereby the poisoning, not of all, but of some one parenchymatous organ ensues, determined by the nature of the poison, and the special qualities of the parenchymatous ele. ments of the different organs. But, if we compare the actions of these common poisons received into the body through the stomach, with the action of aërial poisons inhaled by the lungs, important differences will be found. In the former, the symptoms do not begin with fever, nor is any contagious matter generated in the blood; whereas both these nhenomena are distinguishing features of the latter. Here, then, we have a ground for our argument, that the two parts of the blood have distinct pathological relations; for poisons taken by the stomach exhibit themselves by symptoms which proceed from some local parenchymatous organ, and they do not cause the production of a contagious matter in the blood; whereas poisons inhaled from the atmosphere by the lungs very often do so. Yet both species or classes of poisons act through the medium of the blood.

In 1832, numerous cases of cholera, then epidemic, were treated by saline injections, thrown into the blood in large quantities, not only without hurt to the patient, but in some instances with great apparent benefit. Among many others, there is a very remarkable case reported in the Lancet (vol. ii, 1831 and 1832), in which five gallons of saline liquid were injected by a vein, in four days.

The first injection was to the amount of nine pounds and a half; it was thrown into the circulation in the space of eighteen minutes. A few hours afterwards an additional ten pounds of the saline fluid were injected, with four ounces of albumen. This was at one of the clock on the morning of the 29th May at half-past seven the same morning the injection was repeated to the amount of ten pounds, with the addition of ten grains of sulphate of quinine; at half-past eleven the injection was repeated to the amount of ten pounds. On the second of June, at half-past four in the morning, six pounds and a half of the saline fluid were injected, with six drops of a solution of morphia; and on the 19th of June the patient is reported to have left the hospital, well. 